\title{
LA RECEPCIÓN DE GÖDEL EN ESPAÑA*
}

\author{
Paula OLMOS y Luis VEGA
}

Abstract: Gödel's reception in Spain.

We approach the introduction and repercussion of Gödel's famous theorems of completeness (published in 1930) and incompleteness (published in 1931) from the context of Modern Logic reception in Spain. Spanish reception of these Gödel's results was indeed a troubled and long-drawn-out process from some early and uncertain references and allusions [D. Garcia 1933, 1934], to the first proper statement of his logicomathematical proofs and theorems [M. Sacristan 1964], and finally the translation of Gödel's papers (1930 and 1931) into Spanish at the beginning of '80s.

Key Words: Gödel completeness, Gödel incompleteness, Modern Logic reception in Spain.

\section{Resumen}

Abordamos la introducción y la repercusión de los famosos teoremas de "completud» (publicado en 1930) e "incompletud" (publicados en 1931) de Gödel en el contexto de la recepción de la lógica moderna en España. La recepción hispana de estos resultados de Gödel fue, por cierto, un largo y accidentado proceso a partir de unas tempranas e inciertas referencias y alusiones [D. Garcia 1933, 1934], hasta la primera exposición cabal [Sacristán 1964] de sus pruebas y teoremas logico-matemáticos $y$, en definitiva, la traducción al español de los articulos de Gödel (1930 y 1931) a principios de los años 80.

* Trabajo realizado en el marco del proyecto de investigación BFF 2002-03856. 
Palabras clave: teorema de "completud" de Gödel, teoremas de «incompletud" de Gödel, recepción de la lógica moderna en España.

En la reciente Enciclopedia Oxford de la Filosofia, editada por Ted Honderich (1995) ' ${ }^{1}$, se califica a Kurt Gödel (1906-1978) como «el más grande de los lógicos matemáticos y un audaz y heterodoxo filósofo de la matemática». Puede sorprender que, tras semejante presentación, se despache el artículo sobre Gödel en poco más de una columna. Irónicamente, esta parquedad es un rasgo estilístico de sus propios escritos: escuetos y precisos en lógica, donde parece dotado de la virtud de acertar a la primera; bastante circunspectos en filosofía. Gödel mismo, aunque no dejara de abrigar intereses y preocupaciones filosóficas -en sus últimos años muy intensas-, procuraba rehuir las disquisiciones de los demás y los debates sobre sus impactantes resultados $\mathrm{e}$ ideas.

La obra publicada en vida por Kurt Gödel se reduce a una serie de artículos en los que fue dejando caer sus sucesivos descubrimientos, algunos auténticas bombas de relojería matemática, primicias, otros, de nuevos procedimientos y nuevas perspectivas, al tiempo que ofrecía a los demás la tarea de sacar las consecuencias y de rehacer o desarrollar los sectores de la lógica matemática que se veían afectados por sus métodos, sus teoremas o sus sugerencias.

Así, con un estilo preciso, matemáticamente impecable y nada pródigo en explicaciones que consideraba superfluas, demostró entre otras cosas: la completud de la lógica de primer orden ${ }^{2}$, la incompletud de la formalización estándar de la aritmética - supuesta su consistencia - y la existencia de enunciados deductivamente indecidibles ${ }^{3}$, la indemostrabilidad interna de la consistencia

1 Enciclopedia Oxford de Filosofia, Madrid, Tecnos, 2001, p. 447.

2 Esta demostración constituyó su Tesis doctoral Úber die Vollständigkeit des Logikkalküls (1929) y fue posteriormente publicada con el título «Die Vollständigkeit der Axiome der logischen Funkrionenkalkuilsw en Monatshefte fuir Mathematik und Physik, 37, 1930. La versión inicial se encuentra en la edición dirigida por S. FEFERMAN, Kurt Gödel's Colleted Works, vol. I (1986), pp. 60-101; cf. la versión publicada - que suprime casi todas las referencias y consideraciones introductorias de esa primera versión-, ibd., pp. 102-123. Otra presentación fue su ponencia «Vortrag über Vollständigkeit der Funktionenkalküls" en el Congreso sobre Epistemología de las ciencias exactas (Königsberg, septiembre de 1930); también está recogida en la edición citada, vol. III (1995), pp. 16-29.

3 Este es el que será conocido como "Teorema de Gödel" por antonomasia. Anunciado al final de la ponencia de Gödel en el Congreso de Königsberg, antes citada, también asoma en el curso de una discusión durante el mismo Congreso (cf. CW, I, 200-203) y es objeto de una 
de cualquier sistema que contenga la aritmética computable ${ }^{4}$, la consistencia de la aritmética clásica relativa a la aritmética intuicionista ${ }^{5}$, la consistencia relativa del axioma de elección y de la conjetura del continuo de Cantor ${ }^{6}$ y la posibilidad teórica de lo que podríamos llamar el «tiempo circular" de acuerdo con la relatividad general de Einstein ${ }^{7}$. También desarrolló, en aras de sus

comunicación sumaria de Hans Hahn a la Academia de Ciencias de Viena el 23 de octubre de 1930, "Einige metamathematische Resultate über Entscheidungsdefinitheit und Widerspruchsfreiheit"; en CW, I, pp. 141-3. Su presentación oficial es el artículo "Uber formal unentscheidbare Sätze der Principia Mathematica und verwandter Systeme", enviado a Monatshefte für Mathematik und Physik en noviembre de 1930 y publicado en enero de 1931 (Monatshefte..., 38 [1931], 173-198); en CW, I, pp. 144-195. A veces se ha fundido con On undecidable propositions of formal mathematical systems, Princeton, I.A.S., 1934, que son en realidad apuntes de un curso de Gödel de 1931 tomados por S.C. Kleene y J.B. Rosser; luego fueron revisados por el propio Gödel para una edición de 1965, a la que añadió un Postscriptum (1964); cf. CW, I, pp. 346-71.

4 Este segundo resultado, el llamado "Corolario" del "Teorema" de incompletud, no aparece en los primeros anuncios del teorema en el Congreso de Königsberg, ni en una versión de sus resultados fechada hacia 1931: "Über unentscheidbare Sätzen, en CW, III, pp. 30-35. Pero ya está presente en la comunicación y en el artículo citados en la nota anterior. John von Neumann, matemático húngaro que también acudió a Königsberg y que, al parecer, supo captar de inmediato la importancia del anuncio de Gödel, llegó a este corolario de manera independiente; escribió a Gödel sobre el asunto el 20 de noviembre de 1930, cuando Gödel ya lo había recogido en su propia demostración y enviado para su publicación a Monatshefte.

5 Durante los años 1932 y 1933, Gödel prestó atención a la lógica intuicionista y se dedicó a esclarecer las relaciones entre la matemática intuicionista y la clásica. Un artículo relevante en el primer caso es "Eine Interpretation des intuitionistischen Aussagekalküls", Ergebnisse eines mathematischen Kolloquiums [edit. por K. Menger], 4, (1931-1932, publicado en 1933), 39-40; en CW, I, 300-2, cf. traducción y notas en L. Vega, Lecturas de lógica, Madrid, UNED, 1984 reimp., pp. 301-316. En el segundo caso, "Zur intuitionistischen Arithmetik und Zahlentheorie", Ergebnisse ... [K. Menger, ed.], l.c., pp. 34-38; en CW, I, 286-95.

6 Sobre este tema trabajó entre los afios 1938-40. Halió la consistencia relativa de los dos principios y la expuso en la ponencia "The consistency of the Axiom of Choice and the Generalized Continuum Hypothesis", Proceedings of the National Academy of Sciences, 24, U.S.A., 1938; los apuntes tomados por G.W. Brown en un curso posterior de Gödel sobre este asunto fueron publicados bajo el título The consistency of the Axiom of Choice and of the Generalized Continuum Hypothesis with the Axioms of Set Theory, Princeton, Princeton University Press, 1940 (en CW, II, pp. 33-101).

7 Gödel escribió dos trabajos dando soluciones matemáticas a las ecuaciones de la teoría de la relatividad. El primero, "An example of a new type of cosmological solution of Einstein's field equations of gravitation", Review of Modern Physics, 21, 1949, ofrecia una solución rotatoria y no expansiva del universo que, teóricamente, permitiría el traslado hacia instantes anteriores del tiempo. El segundo, una ponencia presentada en el International Congress of Mathematicians, celebrado en 1950 y titulada "Rotating Universes in General Relativity" (publicada en Proceedings of the International Congress..., Cambridge, MA, 1952), presentaba un nuevo mode- 
resultados lógico-matemáticos, el procedimiento de gödelización, o codificación numérica de expresiones lingüísticas formales, que permite tratar tanto ciertas cuestiones lógicas - - e.g. la cuestión de si una fórmula dada es derivable de otra fórmula-, como cuestiones numéricas - la de si un número dado está en una relación aritmética determinada con otro número-. De paso, abría además nuevas vías de investigación en matemáticas, —e.g. el estudio de las funciones recursivas-, y nuevas perspectivas de desarrollo del análisis lógico - e.g. en las líneas de la autorreferencia de la sintaxis formal dentro del propio sistema o de las relaciones entre la lógica intuicionista y la lógica de la demostrabilidad-.

El caso es que comenzó su carrera con tres demostraciones que, en conjunto, supusieron una profunda inflexión del programa metamatemático de David Hilbert: la primera confirmaba el poder de su aparato lógico elemental, mientras que las otras dos daban al traste con algunas de sus expectativas características —en particular descartaban una axiomatización y formalización completa de la aritmética, capaz de resolver por métodos finitistas cualquier problema de decisión acerca de la derivabilidad de las fórmulas del sistema-, a la vez que refinaban (e innovaban) los métodos formales y probaban su eficacia. Los tres teoremas resultantes -a saber: la completud de la lógica de primer orden (1930), la incompletud de una sistematización de la aritmética en la lógica de Principia Mathematica o sistemas afines y la imposibilidad de demostrar su consistencia dentro del sistema (1931)—, marcaban en suma ciertas posibilidades y limitaciones básicas de los formalismos en uso ${ }^{8}$. No es

lo donde la expansividad del universo impediría tal anomalía. Según S. W. Hawking que prologa ambos trabajos en la edición de las Collected Works de Gödel (II, pp. 190-8 y 208-16 respectivamente), éste sería un modelo más razonable.

${ }^{8}$ David Hilbert (1862-1943), matemático alemán conocido por su axiomatización de la geometría euclidiana (Grundlagen der Geometrie [1899]), se convirtió a principios del siglo XX en uno de los impulsores del formalismo en el que veía el instrumento más adecuado para resolver todos los problemas de fundamentos matemáticos. En 1900, en el Congreso Internacional de Matemáticas de París, enunció una lista de 23 problemas pendientes que habrían de orientar buena parte de la investigación matemática del s. XX; entre ellos destacaba la demostración de la consistencia de la aritmética, empresa que él mismo lideraría más tarde. Los resultados de Gödel acabaron con el optimismo resolutivo del programa de Hilbert y con sus esperanzas de lograr esa prueba por los métodos finitistas que preveía su teoría de la demostración metamatemática. No obstante, Hilbert y sus colaboradores de Göttingen contribuyeron decididamente a la aceptación y difusión de los teoremas de Gödel - e.g. mediante la reelaboración de demostraciones más detalladas-. Por lo demás, estos resultados 
extraño entonces que los historiadores de la lógica hayan empezado a señalar el inicio de la década de los años treinta del siglo pasado como el punto de inflexión más importante en la constitución de la lógica matemática, debido principalmente a la publicación de estos teoremas que no sólo obligaron a replantearse el alcance y el sentido de la lógica sino que, además, fueron el germen de desarrollos ulteriores, como el de la teoría de la computabilidad y sus aplicaciones, entre otras la construcción de lenguajes y de máquinas computadoras — con los considerables efectos por todos conocidos- 9 . Por si fuera poco, Gödel pasó luego a ocuparse de la teoría de conjuntos, con avances sorprendentes en un terreno que llevaba largo tiempo empantanado. Más tarde, casi como divertimento, les aclaró a los físicos más importantes del momento algunas consecuencias matemáticas (¿o físicas?) de sus propias teorías.

De todo ello se desprende que la importancia de la obra de Gödel se aprecia mejor observando el impacto causado sobre el trabajo de otros autores (y generaciones de autores), que limitándose a considerar los logros propios de su genio. De hecho, la recepción de sus obras resulta un campo más interesante, sugerente y extenso de lo que podrían dar a entender el carácter técnico y el tono escueto de sus publicaciones, o la relativa dispersión científica y filosófica de sus intereses ${ }^{10}$. John W. Dawson (1991) y Paolo Mancosu (1999), en particular, han estudiado el momento del primer impacto, la reacción de los lógicos, matemáticos y filósofos que supieron de los descubrimientos de Gödel

de Gödel produjeron a su vez no tanto el abandono completo del programa como la reorientación de algunas de sus líneas: de hecho, la investigación metamatemática posterior ha seguido acusando a lo largo del s. xx los efectos combinados del programa de Hilbert y de los teoremas de limitación de Gödel. Cf. las contribuciones de Sieg, Simpson y Feferman al "Symposium on Hilbert's program" (Washington, D.C.,1885) en The Journal of Symbolic Logic, 53/2 (1988), 337-383.

9 Según Gregory H. Moore, por ejemplo, los resultados de Gödel (1929-1931) marcan el verdadero comienzo de la lógica moderna, vid. la entrada "Logic in the early 20th century" en la Routledge Encyclopedia of Philosophy, London / New York, Routledge, 1989; vol. 5, p. 730. Bastaría recordar los nombres de Gödel, Tarski y Gentzen, o los inicios de teorías fundamentales como la teoría de la computación, la semántica formal o la teoría estructural de la deducción, para calificar los años 1930 de "década prodigiosa".

10 Como ya hemos sugerido, Gödel no aireó sus profundos intereses y sus curiosas ideas filosóficas, mantenidas en su mayor parte inéditas. Alguna muestra de sus manuscritos filosóficos ha visto la luz en el vol. III de $C W$; otras, de los años 50 , se encuentran recogidas y traducidas en la edición de F. Rodríguez Consuegra, Kurt Gödel, Ensayos inéditos, Barcelona, Mondadori, 1994 -vid. su recensión en Éndoxa, 4 (1994), 287-298. También cabe recordar la atención que le merecen Leibniz y Kant, y el interés por Husserl en sus últimos años. 
de primera mano o, al menos, respondieron de manera inmediata a su publicación, así como la progresiva asunción y el desarrollo de sus resultados por parte de la comunidad lógico-matemática internacional ${ }^{11}$.

Por nuestra parte, hemos de constatar la falta de integración de nuestro país en esa comunidad y, en consecuencia, la precaria introducción y la «falta de recepción» efectiva de los teoremas de Gödel en España, por aquellos años. Luego, a pesar de la interrupción y retroceso que en el proceso de modernización en curso supusieron la Guerra Civil y su desenlace - la dictadura nacional católica-, llegó a producirse a lo largo de la segunda mitad del siglo XX una progresiva reintroducción de la lógica matemática en el ámbito de la Universidad española que acabó por normalizar su estudio y, con él, la recepción de Gödel en España en los años 60-70, aunque ésta aún tuviera que pasar por algún trance singular. Por lo demás, no consideraremos los posibles ecos de las contribuciones de Gödel en otros terrenos (e.g. teoría de conjuntos, física teórica).

\section{Kurt Gödel y sus teoremas sobre sistemas formales}

Aquí no podremos detenernos en la vida y milagros de Kurt Gödel, ni en las demostraciones de sus tres teoremas lógico-matemáticos capitales. Hay textos sumamente útiles en ambos sentidos: biografías intelectuales de la calidad de la de John W. Dawson, Logical dilemmas: the life and work of Kurt Gödel (1997); divulgaciones de sus resultados críticos que se han ido refinando a partir de la exposición didáctica pionera de Ernest Nagel y James R. Newman El teorema de Gödel (1958), Madrid, Tecnos, $1970^{12}$. Baste, pues, un breve recor-

11 El internacionalismo de dicha comunidad, en el primer tercio del siglo xx, aunque restringido a Europa y EE.UU., ya era bastante acusado. Alemania, Austria y Polonia albergan los centros principales, pero a ellos acudían lógicos y matemáticos de origen húngaro, escandinavo, etc... Por otro lado, en Francia y Bélgica, los seguidores de Poincaré y los intuicionistas formaban el contrapunto a la escuela formalista de Hilbert. Gran Bretaria segula siendo la cuna de los Principia Mathematica y pronto tendrá un papel relevante en el desarrollo de la teoría de la computación gracias a Turing. Por su parte, EE.UU. comenzó en seguida a recibir de manera transitoria o definitiva a muchos de estos lógicos centroeuropeos - cuya diáspora fue creciendo en los años 30 conforme se extendía la amenaza nacionalsocialista hitleriana-, aparte de desarrollar su propia escuela (Alonzo Church). Todos ellos compartían regularmente sus resultados, celebraban congresos, coloquios y reuniones de todo tipo en un ambiente de efervescencia similar al que, por los mismos años, reinaba en el mundo de la física.

12 Las noticias de Dawson pueden complementarse con las aportadas por uno de los más directos interlocutores y estudiosos de Gödel, Hao Wang (1987), Reflexiones sobre Kurt Gödel, 
datorio informal de los teoremas que facilite la discusión en torno a su introducción y recepción en España.

Se decía que un sistema axiomático es consistente si todas sus proposiciones deducibles son verdaderas en su dominio temático de interpretación y, mạs precisamente, no cabe deducir en el sistema una contradicción: una proposición y su negación; es decir, el sistema no admite ninguna proposición ' $\alpha$ ' que sea tanto demostrable $-\mathrm{y}$ pueda darse una deducción de ' $\alpha$ '-, como refutable $-\mathrm{y}$ pueda darse una deducción de 'no $\alpha$ '- Esta idea se generaliza en un sistema formal en el sentido de que toda fórmula derivable mediante su aparato deductivo es una fórmula válida, verdadera en cualquier dominio y bajo cualquier interpretación del sistema. Por otro lado, se decía que un sistema formalizado es semánticamente completo si todas sus fórmulas válidas son deducibles en el sistema. La consistencia asegura la corrección del sistema; la completud, su suficiencia. Pues bien, el teorema de completud de Gödel (1930) establece que el sistema formal del calculo funcional restringido, i.e. un sistema correspondiente a lo que hoy entendemos como lógica estándar de primer orden, es semánticamente completo (vollständig): todas sus fórmulas válidas son deducibles, cada una de ellas - posiblemente infinitas - sería la conclusión de una deducción realizable conforme a la reglas de derivación del sistema. (La prueba de la consistencia de un sistema de esta clase ya había sido publicada por Hilbert y su colaborador Ackermann en 1928.)

A su vez, el teorema de incompletud (1931) considera un sistema axiomático formal S compuesto por la lógica de Principia Mathematica —una lógica no ya de primer orden, sino de orden superior-y por la aritmética de Peano. La completud, en este contexto, toma un sentido deductivo: un sistema se dice completo si y sólo si, para cualquier proposición formulable en su lenguaje, puede deducir dicha proposición o su negación. Pues bien, Gödel establece que en el lenguaje de cualquier sistema $S$ de este tipo puede construirse una fórmula proposicional ' $\beta$ ' que, en su interpretación natural, dice de sí misma que no es deducible en $S$, y es tal que, si $S$ es consistente, ni es demostrable ni es refutable en $S$ - es decir, ni ' $\beta$ ' ni su negación son derivables en $S$-, de

Madrid, Alianza, 1991. Por otro lado, en el c. 5 de Jesús Mosterín, Los lógicos, Madrid, Espasa, 2000, pp. 219-285, se encuentra una presentación accesible. Y, en fin, en Douglas R. Hofstadter (1979), Gödel, Escher, Bach: un eterno y grácil bucle, Barcelona, Tusquets, 1998, pueden verse proyecciones e ilustraciones de los resultados de incompletud de Gödel en diversos ámbitos culturales. 
modo que resulta una proposición indecidible (unentscheidbar Satz) en S. Por lo tanto, $S$ es un sistema incompleto. Es, más aún, incompletable pues la adopción de ' $\beta$ ' como un nuevo axioma de $S$ no podría evitar la construcción ulterior de otra proposición con sus mismas características y consecuencias en el sistema así ampliado. Por otro lado, como ' $\beta$ ' dice de sí misma que no es deducible, resulta verdadera considerada desde fuera del sistema de mudo que la proposición ' $\beta$ ', al cumplir su no-deducibilidad declarada, será quizá sorprendente pero no paradójica.

Como corolario de este resultado, se desprende el segundo teorema de incompletud de Gödel (1931), que viene a sentar una limitación ulterior: si S es consistente, dicha consistencia no será demostrable con los recursos propios del sistema. A la luz de ambos teoremas de incompletud, cabe concluir que una teoría así formalizada de la aritmética clásica $\longrightarrow$ de cualquier extensión que incluya esta aritmética - no puede ser a la vez axiomatizable, consistente y completa: si es una teoría axiomatizable y consistente, no puede resultar completa y, por añadidura, no estará en condiciones de probar su propia consistencia. (La consistencia de la aritmética clásica sería demostrada ulteriormente por Gentzen (1936), pero con recursos distintos de los considerados propios de un sistema del tipo de $\mathrm{S}$, como el uso de una inducción matemática transfinita.)

La obtención de estos teoremas de incompletud descansa en dos procedimientos metódicos especialmente notables: uno es la "gödelización" ya mencionada; el otro, la autorreferencia sintáctica, la construcción de proposiciones bien formadas capaces de referirse correctamente a sus propiedades formales, e.g. en el sentido de decir de sí mismas que son indemostrables ${ }^{13}$.

\section{Contexto de presentación y recepción de los resultados}

Los resultados de Gödel tuvieron la suerte de producirse en un medio que no tardó mucho en hacerse cargo de ellos (aunque fuera con distintas actitu-

13 Esta autorreferencia formal o sintáctica difiere sustancialmente de una semántica como la de la proposición que dice de sí misma que es falsa. Por un lado, se ve libre de paradojas tradicionales como la del "mentiroso" (el que dice "miento", ¿miente o dice verdad?); por otro lado, el concepto semántico de verdad (o falsedad) no es definible ni capturable dentro de un sistema formal, a diferencia de los conceptos sintácticos formalmente definidos como los de demostración o demostrable — cf. sus definiciones respectivas, 45 y 46, en Gödel (1931)—. 
des $\left.{ }^{14}\right)$, ya que afectaban directamente al trabajo de muchos lógicos y su actualidad no podía ser mayor. Efectivamente, el singular y novel Gödel no tuvo más remedio que convertirse en el centro de atención de la comunidad lógico-matemática y para ello se encontraba quizás en el mejor sitio posible: en Viena.

Alumno del matemático Hans Hahn, su director de tesis y, con él, asiduo de los círculos lógico-matemáticos vieneses como el de Friedrich Schlick o el coloquio de Karl Menger, Gödel, tras un somero anuncio en el Congreso de Epistemología de Königsberg el 6 de septiembre de 1930, pudo presentar sus resultados al poco tiempo ${ }^{15}$ a un grupo amplio que compartía sus intereses y que, además, estaba bien comunicado con otros círculos lógico-matemáticos. Fueron muchos los que en seguida se interesaron por su descubrimiento de las limitaciones de los sistemas lógico-matemáticos disponibles, sobre todo una vez pasado el trámite de ese Congreso donde la trascendencia del anuncio se le habría escapado a la mayoría de los asistentes, debido, quizá, a lo inesperado de su presentación — su asunción inmediata habría barrido el suelo bajo los pies de buena parte de las ponencias restantes, en especial las de tinte formalista o logicista-. De estos primerísimos momentos se suele comentar el olfato de John von Neumann, el primer entusiasta que quedó impresionado por el hallazgo y trató de extender la noticia ${ }^{16}$, por contraste con la actitud de Rudolf Carnap que, pese a conocer algo del asunto antes incluso del propio Congreso ${ }^{17}$, no consideró oportuno alterar sus posiciones previas, ni enfren-

14 Una de las motivaciones de Dawson (1991), “The Reception of Gödel's Incompleteness Theorems", es tratar de refutar la idea tradicional de que el teorema de incompletud y su corolario fueron inmediatamente aceptados por todo el mundo, matizando las distintas actitudes mostradas por la comunidad lógico-matemática. La verdad, sin embargo, es que el proceso de recepción fue más rápido de lo esperado por el propio Gödel, gracias a las redes de comunicación existentes y a ciertos entornos especializados como el de Göttingen.

15 En el Círculo de Schlick, el 15 de enero de 1931; en el Coloquio de Menger, una semana después, el 22 de enero.

16 Von Neumann comentó los resultados de Gödel a su vuelta de Königsberg, en Berlín, en el círculo de Reichenbach, donde, entre otros, conocieron la noticia Hempel y Herbrand. Este último escribió a Gödel y se interesó por la teoría de las funciones recursivas. Von Neumann sería el responsable de la invitación de Gödel por parte del I.A.S. de Princeton, donde acabó desarrollando su carrera a partir de los años 40 .

17 Gödel se reunió con Carnap y algún otro el día 26 de agosto de 1930, y también tres días después, en el café Reichrat, de Viena, precisamente para hablar del Congreso y sus respectivas ponencias. 
tarse al cambio radical que se cernía sobre la discusión a la hora de publicar las actas del encuentro ${ }^{18}$.

Ya hemos mencionado el efecto desarmante de los resultados de incompletud de Gödel sobre ciertas expectativas del programa de Hilbert. En este contexto cobra relieve la correspondencia entre Gödel y un colaborador directo de Hilbert, Paul Bernays, quien, tras haber planteado algunas dudas iniciales al conocer los resultados, acabó reconociendo pronto la exactitud de las pruebas. Hilbert y Bernays las rehicieron e incorporaron luego estos resultados en su tratado sistemático en dos volúmenes $(1934,1939)$ Die Grundlagen der Mathematik. En este marco, un cierto factor de distorsión en la recepción de los resultados fueron las secuelas del ambiente creado por las antinomias lógico-matemáticas: por un lado, alguna de ellas, e.g. la de Richard, podría haber inspirado el proceder de Gödel (1931); pero, por otro, propiciaron luego su confusión con las temidas paradojas asociadas a la autorreferencia semántica o al uso subrepticio de funciones no predicativas ${ }^{19}$.

Por otra parte, la escuela rival del formalismo, el intuicionismo, al que los teoremas de incumpletud parecían dar la razón en su escepticismo sobre la significación y alcance de la lógica (meta)matemática ${ }^{20}$, no iba a asimilar fácilmente una prueba rigurosamente formalista como la de Gödel. En trabajos posteriores, como ya sabemos, Gödel se ocupó de la matemática intuicionista a la que no era en absoluto hostil, demostrando la compatibilidad y consistencia relativa de la misma respecto de la formulación clásica.

18 La hipótesis de Dawson de que Carnap no comprendió bien a Gödel ha sido contestada por Mancosu (1999), que, a la luz de una serie de cartas de Carnap dirigidas a von Neumann y Reichenbach, trata de mostrar que Carnap tan sólo quería salvar la situación y se había tomado la publicación de las actas como una ocasión para recoger el estado de la cuestión en un momento determinado (previo a Gödel) sin tratar de encontrar un punto de solución final que, probablemente, no creía que se hubiera alcanzado. De hecho, en un primer momento, hasta el propio Kurt Gödel y el entusiasta Von Neumann mantuvieron una cierta esperanza de "revisión" de los resultados como se desprende de la carta que este último escribió a Reichenbach (reproducida por Mancosu).

19 Por ejemplo, a un lógico matemático tan competente como el polaco Leon Chwistek le costó algún tiempo sobreponerse a la primera impresión de que Gödel (1931) envolvía un uso paradójico de la impredicatividad. Y, fuera del ámbito lógico matemático, su asimilación a la antinomia del "mentiroso" causó algún estrago.

20 Tanto Gödel como sus inmediatos interlocutores asumieron que el intuicionismo salía indemne de los resultados obtenidos ya que la matemática intuicionista ni siquiera admitía en principio la importancia de la formalización y el concepto de sistema formal en el que se basaban las pruebas. 
Con respecto a la originalidad de los resultados, ya hemos indicado que J. von Neumann llegó a obtener, de manera independiente, el "corolario de Gödel», aunque en vista de las circunstancias decidiera no reclamar su autoría. Mayor motivo para arrogarse una prioridad genérica del planteamiento, en su conjunto, podría haber tenido Emil Post: casi diez años antes que Gödel, ya había sugerido que sus propios métodos podrían dar lugar a proposiciones indecidibles en el sistema de Principia Mathematica, inquietud que le llevó a interesarse por los problemas de indecidibilidad aunque de manera menos «sistemática", según él mismo confiesa, y a tratar con la noción de indecidibilidad absoluta. Lo cierto es que Emil Post aclamó el trabajo de Gödel como una prueba precisa y definitiva de sus propias ideas, tanto en su correspondencia como en un largo trabajo de 1941, "Absolute unsolvable problems and relatively undecidable propositions. Account of an anticipation» -luego publicado por M. Davis, ed. The undecidable, New York, Raven Press, 1965, pp. 340433. El problema de la «indecidibilidad absoluta», que Gödel no contemplaba -fue gracias a su precisión sistemática, su lucidez lógica y su rigor formal como pudo establecer sus resultados-, también había preocupado a Paul Finsler, quien reclamó a Gödel la prioridad del descubrimiento mencionando su trabajo de 1926 sobre el asunto. Pero el ensayo de Finsler dejaba bastante que desear en cuanto a precisión y cuidado en el manejo de los niveles lingüísticos y del aparato formal, y su reclamación carecía de base -cf. Van Heijennort (1967), pp. 438-440.

Otros, en cambio, se opusieron a Gödel por discrepar radicalmente de su planteamiento; entre ellos, el matemático Ernst Zermelo quien, ya en septiembre de 1931, en un encuentro de la Sociedad Matemática Alemana en BadElster, criticó el proceder gödeliano precisamente por "finitista»; Zermelo confiaba en las posibilidades y ventajas de no atenerse a las restricciones metódicas de este tipo recomendadas por el programa de Hilbert —cf. Dawson (1895)—.

Pero las críticas más virulentas a las pruebas de Gödel (1931) aparecieron algo más tarde y fuera de este marco: en particular, las de Chaïm Perelman (1936), Jerzy Kuczynski (1938) y Marcel Barzin (1940). La más notoria, en su momento, fue la de Perelman, criticada a su vez por Grelling y Helmer, y definitivamente desarmada por Kleene (1937). Perelman había creído encontrar una antinomia en la prueba de Gödel (más bien en su exposición informal introductoria); pero, en realidad, había sido muy poco cuidadoso en su tratamiento de los niveles lingüístico y metalingüístico de la prueba y se había dejado guiar por una versión intuitiva que ignoraba o pasaba por alto el sutil recur- 
so a la autorreferencia sintáctica y a la gödelización, un recurso inconfundible con la autorreferencia semántica o con otros generadores de paradojas por reflexividad. El cargo de antinomia o contradicción interna, amén de unas confusiones y unos descuidos análogos, reaparecerán luego en algún crítico español rezagado (cf. $\$ 4.2$, infra).

En la vertiente positiva de la recepción, los resultados de limitación de Gödel adquirieron mayor generalidad y extensión gracias a los propios trabajos posteriores de Gödel (1934) y a nuevas contribuciones de Church (1936), Rosser (1936) y Kleene $(1936,1943)$. Por otro lado, ya hemos mencionado que los resultados de Gödel facilitaron el desarrollo del trabajo lógico en problemas de decisión y computabilidad en que se ocuparon especialmente Alonzo Church (quizá el heredero más directo de este legado) y Alan Turing. La asociación de las limitaciones de los sistemas formales de Gödel con las limitaciones de las máquinas lógicas de Turing, y la asunción de ambos desarrollos lógicos, el formal y el mecánico, como correlatos técnicos de la noción informal de calculabilidad o de método efectivo no sólo fueron el germen de la teoría de la computabilidad, sino el motivo de proyecciones y extrapolaciones sobre las relaciones mente-máquina y sobre los problemas de la inteligencia artificial. Hoy todavía sigue abierta la cuestión de si la creatividad de la mente humana desborda la capacidad de cualquier sistema o máquina lógica, y la discusión acerca de las posibilidades y límites de la simulación de la inteligencia por medio de artefactos de computación, debates en los que suelen traerse a colación los teoremas de Gödel (1931) - a veces sin caer en la cuenta de que sus resultados descansan en un supuesto de consistencia que no se sabe muy bien cómo precisar en el caso general de "la mente humana»- Ahora bien, para auténticos despropósitos, ahí están los cometidos por ciertos sociólogos que han dado en hablar de las limitaciones y constricciones de los sistemas sociales en términos "gödelianos" hasta el punto de apañarse una suerte de "teorema Gödel-Régis Debray" a estos efectos ${ }^{21}$. Por fortuna, los resultados de Gödel pueden mostrar su plena significación lógica, matemática, científica y filosófica, sin necesidad de morir de éxito en el intento.

21 J.BOUVERESSE (1999), Prodigios y vértigos de la analogia, Buenos Aires, Zorzal, 2001, denuncia este abuso (cf. pp. 91-106 en especial); es un episodio más de nuestra historia reciente de atropellos e imposturas intelectuales, vid. A. SOCAL y J. BRICMONT (1998), Imposturas intelectuales, Barcelona, Piados, 1999, pp. 175-9. 


\section{Primeros ecos de Gödel en España (décadas1930-1950)}

Esbozado el perfil general de la recepción de los resultados de Gödel, como telón de fondo y marco de comparación, consideremos ahora su suerte en España. Dentro de los límites de una exploración inicial como la que representa el presente artículo, habremos de limitarnos a comentar ciertos casos singulares y especialmente ilustrativos.

\subsection{David García (Bacca) y el despertar de la nueva lógica en España}

A principios del siglo veinte, la lógica sobrevivía en España como una rutina escolar estancada en medio del desinterés general, no desmentido por su condición de disciplina obligada en los planes de enseñanza oficial, secundaria y universitaria - para los estudiantes de Filosofía y Letras e incluso de DerechoConfinada en el ámbito de la filosofia, era un cajón de sastre en el que se mezclaban unas rutinas lógicas tradicionales -e.g. la tradición "aristotélica" del silogismo- con temas epistemológicos y retales metodológicos más o menos modernos. Desde los años 1890 venían llegando algunas noticias sueltas sobre las nuevas lógicas europeas y americanas (la lógica simbólica de la línea BooleSchröder, la lógica matemática de Peano y su escuela, el círculo de Peirce), procedentes inicialmente de algún matemático curioso; pero no llegan a prender por diversas circunstancias y motivos, entre otros la falta de suelo nutricio - de una base conceptual de integración o una base técnica de asimilación-, y la ausencia de ecos intelectuales o institucionales. Las dos primeras décadas del siglo siguen asistiendo a alguna que otra iniciativa individual, aislada, que tampoco podrá salvar el abismo existente entre esas nuevas lógicas y las ideas y prácticas establecidas en nuestros medios académicos, tanto filosóficos como matemáticos ${ }^{22}$. Por fortuna e inopinadamente, puesto que los intereses de modernización y aculturación de nuestra llamada «Edad de Plata» se movían lejos de esta área lógico-matemática -aún en su Edad de Bronce-, con los años 30 aparecen, en catalán, los primeros signos ciertos de la introducción de la nueva lógica en

22 Cf. al respecto L. VEGA, "La lógica en España (1890-1930): desencuentros", Teorema, XX/1-2 (2001), 21-38; “Ventura Reyes Prósper y la introducción de la nueva lógica en España”, Asclepio, LIV/2 (2002), 181-209. 
España. El «introductor» por antonomasia es un joven claretiano, David García - luego reconocido como una de nuestras autoridades intelectuales en el exilio: Juan David García Bacca-, que vuelve de su peregrinaje por varias universidades europeas con un bagaje de conocimientos de física teórica, matemáticas y lógica, y una seria amenaza de crisis personal e intelectual. Hacia 1930, según recordará más tarde, sus contactos con los transfinitos cantorianos, a través de Fraenkel (1928 3. ${ }^{a}$ edic.), Einletung in die Mengenlehre, y con el cálculo lógicomatemático, a través de Hilbert y Ackermann (1928), Grundzüge der theoretischen Logik, están repercutiendo violentamente en su formación escolástica inicial 23. El caso es que las primeras referencias a los teoremas de Gödel publicadas en España traen la firma de David García en sendas obras de 1933, Assaigs moderns per a la fonamentació de les matemàtiques, y 1934, Introducció a la logistica, el primer tratado de presentación de la nueva lógica en España.

Los Assaigs no mencionan Gödel (1929-30), pero sí hacen referencia y mención bibliográfica de Gödel (1931) en el contexto de la crítica del programa de Hilbert. La referencia discurre en los términos: «en qualsevol sistema lògic que enclogui una aritmética poden ésser bastides fórmules aritmètiques la validitat o invaliditat de les quals és indiscernible amb els mitjans lògics", para añadir que si esta demostración se confirma o afianza, el formalismo verá muy menguadas su importancia y sus pretensiones (p. 46). Alegan, por otro lado, la imposibilidad de demostrar, en cualquier sistema lógico-matemático, que el sistema en su conjunto es no contradictorio o compatible (p. 46). Pues no cabe demostrar la proposición "el sistema A, en cuanto sistema, es internamente no contradictoriom con los medios demostrativos del propio sistema, en razón de que según la teoría de los tipos -admitida por todo el mundo como vía de solución de las paradojas- dicha proposición es de un tipo superior al de las proposiciones del sistema en cuestión. En suma, reiteran los Assaigs,

23 El propio García Bacca se ha forjado una barroca teoría biográfica, compuesta por sucesivos choques filosóficos y científicos contra fondos de creencias previas, en su "Autobiografia intelectual de Juan David García Baccan, Anthropos, 9 (1982), 4-10. Véanse también sus Confesiones, Barcelona, Anthropos, 2000, donde da cuenta de su encuentro con la nueva lógica: «En 1929, antes de la vuelta a España, pasé por la librería para ver las últimas obras. Me hallé con la novísima edición de "Grundzüge der theoretischen Logik" (1928), de H. (sic) Hilbert y W. Ackermann. No conocía aún "Principia Mathematica» de Russell-Whitehead, tres vol., 1910-1913. Obra monumental, si esta denominación se la aplica justicieramente a una obra impresa. La compré y durante el largo viaje la estudié. Algo nuevo, originalísimo, respecto de la lógica aristotélico-tomista” (p. 43). 
"és impossible demostrar la compatibilitat de tot el sistema logic en conjunt per mitjans logics. Si creiem les matemàtiques una part de la lógica, és imposible demostrar la compatibilitat interna de les matemàtiques, com a sistema, pels mitjans lógics emprats en llur construcció, i si n'emprem d'altres, al capi i a l'últim ens trobarem davant la impossibilitat de demostrar amb mitjans lògics la compatibilitat total de la lògica. Si volem, doncs, demostrar-la, ens cal sortir del camp lògic, és a dir, recórrer a mityjans fonamentats en la significació extralògica i arribarem al camp de la criteriologia i de la teoria del coneixement" (p. 47).

Esta interpretación es sintomática, en parte, de la hegemonía de Principia Mathematica y la teoría de los tipos en la lógica matemática de los años $30 \mathrm{y}$, en parte, de la irregular formación autodidacta de nuestro autor. No se detiene a distinguir entre la lógica de primer orden y la de orden superior en las alusiones a la consistencia o no contradictoriedad del sistema de la lógica, a pesar de su supuesto conocimiento de Hilbert y Ackermann (1928); ofrece una razón de la imposibilidad de demostrar la consistencia del sistema lógico matemático, en su conjunto, que no tiene que ver con las pruebas de Gödel (1931) - si acaso, se opondría a su proceder característico de gödelización y representación de la sintaxis en el sistema mismo-; y si, en general, David García acusa algunas dificultades de comprensión del nuevo horizonte metamatemático, en particular no cabe atribuirle un conocimiento directo del artículo de Gödel (1931).

La Introducció, al margen de sus reconocidos méritos como exposición relativamente cumplida de la "logística» pre-gödeliana, y de su contribución a la introducción de la nueva lógica en España, está mejor informada - e.g, en el terreno de la (meta)lógica proposicional-, avanza algunas nociones metateóricas básicas y parece familiarizada con la estrategia fragmentaria de Hilbert, con su estudio de las propiedades metamatemáticas de los subsistemas que conforman el cuerpo general de la logística. Pero la Introducció no es muy prometedora en el aspecto concreto de la recepción de los teoremas de Gödel. Por ejemplo, tras distinguir entre la suficiencia o integritat semántica de un sistema - la capacidad de deducir todas sus fórmulas verdaderas-, y su compleción sintáctica - de modo que la adición al sistema de una fórmula no deducible deriva en una contradicción-, David García escribe: "Ambidues integritats posseeix el cálcul proposicional, segons Bernays-Hilbert. S'ha demonstrat, pèro, que el sistema dels six axiomas no posseeix la qualitat d'integritat” (l.c., II, p. 147). El «sistema dels six axiomes» es un sistema del cálculo fun- 
cional de primer orden ${ }^{24}$. Hilbert y Ackermann (1928) habían reconocido que este sistema no es completo sintácticamente, mientras que su suficiencia en el sentido semántico era una cuestión por resolver. Su solución positiva fue precisamente el resultado publicado por Gödel (1930), texto a todas luces ignorado por la Introduccid pues no lo recoge siquiera en la bibliografía. Ahí, en cambio, aparece Gödel (1931). Pero nuestro autor, al suponer la no «integridad" — sin mayores precisiones - del sistema de los seis axiomas, tiende a confundir la lógica de primer orden y la de orden superior - con la inclusión de la aritmética-. La confusión, propiciada por sus referencias genéricas al "sistema de la logística", ya había asomado un poco antes, cuando David García aseguraba que la compatibilidad o consistencia del sistema total de la logística aún no se había podido demostrar (ibd., p. 145). Del contexto se desprende que este sistema es una vez más el de "los seis axiomas", de primer orden; sin embargo, Hilbert y Ackermann (1928) habían probado la consistencia de un cálculo de este tipo, al tiempo que consideraban el caso del cálculo funcional extendido o de orden superior como una cuestión abierta; y, por otra parte, Gödel (1931) había establecido como corolario o teorema derivado del de "incompletud" que la consistencia de sistemas logísticos como el de Principia Mathematica -i.e. la formalización de la aritmética de Peano en su lógica de orden superior- era indemostrable con los medios propios del sistema. Lo cual confirma la sospecha de que nuestro autor no distinguía bien uno y otro orden de formalización y sistematización.

Otra muestra análoga podría ser su consideración de la cuestión general de la decidibilidad: a este respecto, nuestro autor declara: «malauradament, la logística total no ha trobat encara el método convenient» (ibd., p. 148). Esta cuestión ya había merecido su atención en el vol. I, pp, 184-194, donde había consignado la decidibilidad de la lógica proposicional y apuntado algunas soluciones parciales en el caso de la lógica cuantificacional o "conjuntual». Pero ahora no deja de ser curioso que, aun citando a Gödel (1931) en la bibliografía, pase por alto la existencia de fórmulas indecidibles en la logística de Principia Matemática, como precisamente la fórmula crítica que afirma su propia indemostrabilidad, fórmula tal que ni ella - aun siendo verdadera - , ni su negación resultan deducibles en el sistema en el supuesto de que éste sea consistente.

${ }^{24}$ Estos axiomas son los cuatro de la lógica proposicional (según Hilbert-Bernays): '( $\left.p \vee p\right)$

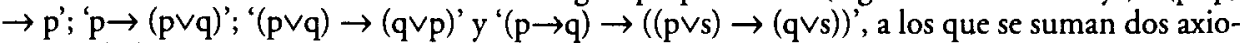
mas de la lógica cuantificacional - o "conjuntual», en términos del autor-: ' $(x)[F(x) \rightarrow F(y)]$ ' $y^{\prime} \mathrm{F}(\mathrm{y}) \rightarrow(\mathrm{Ex}) \mathrm{F}(\mathrm{x})$ '. Véase o.c., I, $\$ 22$, pp. 160-1. 
En suma y seguramente por falta de trato directo con el texto, es una lástima que la Introducció de David García no haya sacado mejor partido de Gödel (1931). Máxime si se tiene en cuenta que la quinta y última parte del libro se dedica al examen y la valoración de la logística en su conjunto, a partir de una cuestión capital: «¿Hem formalizat tota la lógica?», que se desplegará en tres preguntas:

"¿Hem axiomatizat la logística complint las tres conditions de compatibilitat, independencia i integritat dels axiomes? ¿Hem aconseguit de formuler simbòlicament, i mitjançant definicions implicites, sense recórrer a la significació, les lleis i processos tots de la logística? Finalment, ipertanyen a la logística tots els mitjans de deducció i axiomes emprats?? (II, p. 143).

Las respuestas del autor tenderán a ser negativas, especialmente críticas del programa formalista de Hilbert. Pero, en su actitud, las consideraciones filosóficas -e. g., la contemplación de una lógica trascendental, plurivalente e integrada en una suerte de saber universal- pesan más que el estudio atento de los resultados técnicos de Gödel. Anotemos este rasgo pues no dejará de ser sintomático de la utilización de Gödel por parte de algunos de nuestros filósofos que se permitirán referencias y versiones de "el teorema» de Gödel (¿sería una impertinencia preguntar cuál?), sesgadas por cuestiones e intereses de orden filosófico y poco respetuosas con los detalles y procedimientos técnicos lógico-matemáticos.

El propio García Bacca tuvo ocasión años más tarde de volver a referirse a Gödel (1931) en sendas reseñas de Nagel y Newman (1958) Gödel's proof, y de la edición inglesa (1962) del artículo de Gödel prologada por R. Braithwaite -reseñas publicadas en Revista Nacional de Cultura (Caracas), 155 (1962), 169-171. Por entonces ya está al tanto de la gödelización y de algunos supuestos sintácticos de la prueba; pero insiste en sus intereses especulativos y en sus preocupaciones filosóficas hasta el punto de recomendar una orientación en esa línea que ayude a comprender "el meollo y alcance de la prueba" (p. 170), y de interpretar su resultado como la refutación del ideal husserliano, fenomenológico, de una ciencia universal (p. 171).

\subsection{El exilio exterior como apertura: José Ferrater Mora}

Desde 1936, la mayoría de nuestros representantes intelectuales más valiosos o prometedores tuvieron que buscarse la vida en el exilio, García Bacca 
entre otros. Uno de los que quisieron impulsar la aculturación lógica en espanol fue Ferrater Mora. Todo hace suponer que lo consiguió gracias a que el exilio le proporcionó la oportunidad de instalarse en EE.UU. y de entrar en contacto con el ambiente de la lógica y la filosofía analítica de los años 40 y 50 .

En este sentido, tiene interés estudiar la evolución de su Diccionario de Filosofia cuya primera edición, publicada en La Habana, data de 1941. Ferrater Mora, filósofo que provenía de la "Escuela de Barcelona" y del ambiente creado en torno a su Universidad Autónoma durante la República, fue capaz de componer un Diccionario de Filosofia de gran influencia en todo el ámbito hispanohablante que, en posteriores ediciones y al mediar el siglo, acabaría llegando a España e informando de nombres, nociones y aspectos de la filosofía del s. xx poco tratados aquí.

Por lo que a la lógica concierne, el gran cambio entre las distintas ediciones de Diccionario se produjo entre la segunda (Santiago de Chile/México, 1944), muy similar a la primera, y la tercera (Buenos Aires, 1951), publicada tras una estancia de año y medio en Pennsylvania (Bryn Mawr College). El propio autor comenta, en el prólogo, que los principales cambios y el aumento bastante espectacular del volumen se debían a la renovación que había tenido que introducir en el campo de la "lógica actual y la teoría de la ciencia», al que había llegado a través de su familiarización progresiva con la filosofía en circulación en EE.UU ${ }^{25}$.

Así, en la edición de 1944, no encontramos artículos sobre Frege ni sobre Cantor, Peano o Gödel. La entrada "PARADOJA" no remite a ningún problema lógico ni matemático, sino que contiene una definición general de tipo lingüístico. En el artículo sobre "MATEMÁTICA», se nombran las tres escuelas de "la época actual", formalista, logicista e intuicionista, sin que se haga referencia alguna a la "crisis de fundamentos". El apartado dedicado a la "LOGICA" contiene una historia de sus distintas concepciones de carácter

25 Recordemos que, en Argentina y en español, ya existía una presentación de Lidia Peradotto, La logistica, Buenos Aires, Imp. de la Universidad de La Plata, 1925, y circulaban noticias sobre la nueva lógica y filosofía de la matemática, como las difundidas por Fausto Toranzos en este caso es curioso que su notable y conocida Introducción a la epistemologia y fundamentación de la Matemática, Buenos Aires, Espasa-Calpe, 1943, 1949 2. a edic., haga referencia a los resultados críticos de incompletud de Gödel (1931) remitiendo al lector al artículo de Gödel (1930) sobre completud, resultado este que no recibe en cambio la menor atención. 
muy general, desde Aristóteles hasta Husserl, sin mencionar las obras de Frege ni de Russell; dentro de su orientación tradicional lógico-filosófica, tiene un peso especial la idea de la lógica como "sistema de pensamiento» en la línea fenomenológica de Pfänder. La nueva lógica matemática sólo aparece fugazmente en las entradas "LOGICISMO" y "LOGISTICA». En resumen, es una obra de referencia que recoge múltiples aspectos de la filosofía coetánea y donde la nueva lógica aparece nominalmente tratada, con un espacio propio, pero sin que su contenido y su sustancia, o su aportación a la clarificación y codificación de determinados conceptos lógicos, filosóficos o matemáticos se vean reconocidas y apreciadas.

Todo ello se reestructura, como ya hemos indicado, en la edición de 1951. Ahora, por fin, encontramos a nuestro protagonista Kurt Gödel con su propia entrada aunque ésta remite a otras cuatro, dos antiguas convenientemente reelaboradas «MATEMÁTICA» y "VIENA (Círculo de)» y dos completamente nuevas "CONTINUO" y "METALENGUAJE». Es, precisamente en esta última donde se entra a comentar la "Memoria» de Gödel de 1931, haciendo hincapié en el llamado corolario o segundo teorema (quizá más accesible). No falta otra mención de los resultados de Gödel en el artículo dedicado a «CONSISTENCIA" que añade, sobre la edición anterior, un largo apartado matemático en el que se introduce su sentido en el «sistema formal de Hilbert». También se ofrece bibliografía en torno a Gödel a lo largo de la obra. Este giro reviste importancia porque, a pesar de revelar un conocimiento no adquirido en España ni realmente introducido en el sistema educativo de nuestro país, el Diccionario de Ferrater Mora fue una de las vías por las que pudo llegar a penetrar en dicho ámbito: venía en castellano y en un vehículo -como es una obra general de referencia - disponible para un público mucho más amplio que el de un trabajo monográfico.

Por otro lado, Ferrater tampoco se ahorró dos monografías sobre lógica. Una es un manual compuesto en colaboración con Hugues Leblanc ${ }^{26}$, (1955) Lógica matemática, que tuvo cierta fortuna entre los interesados por la lógica en España a finales de los años 50 y principios de los 60, a la vez que les ocasionaba algunos quebraderos de cabeza por ciertas omisiones y oscuridades. Incluía un capítulo dedicado a la "Metalógica", donde se daban las definiciones

26 Hugues Leblanc ya habia publicado An Introduction to Deductive Logic, New York, Wiley \& Sons, 1955. Ambos coincidieron en el Bryn Mawr College de Pennsylvania. 
correspondientes a las propiedades metalógicas de los sistemas formales. Los autores presentan el primero de nuestros teoremas de Gödel, la completud semántica del cálculo cuantificacional elemental de 1929-30 (p. 178) y, a continuación (pp. 179-182), los resultados negativos respecto del cálculo cuantificacional superior, con detalles sobre el proceso de gödelización, cuya prueba se fecha en "1933"; faltan las menciones bibliográficas pertinentes de Gödel (1930) y Gödel (1931) —en su lugar, la 2.a edic. de 1962 cita una versión mexicana (1959) del popular Nagel y Newman, El teorema de Gödel-. Por lo demás, su versión del teorema de incompletud no deja de ser original: el teorema dice que una fórmula del cálculo de $P M$ «es susceptible de prueba si y sólo si es falsa» (versión mantenida en las ediciones de 1962 y 1965). También mencionan el doble carácter completo e indecidible del cálculo de primer orden, pero por referencia a Church «1934» (a la prueba publicada por Church en 1936, cabe suponer). La otra monografía, (1957) Qué es la logica, quiere ser una presentación general de la materia para filósofos: lo cierto es que todavía se resiente de una transacción y transición entre la lógica filosófica tradicional y la lógica matemática moderna, amén de que resulta demasiado genérica y sumaria para incluir resultados metalógicos o planteamiento técnicos.

En fin, las sucesivas ediciones del Diccionario de Filosofia $(1956,1964)$ fueron ampliando la obra en muy diversos sentidos aunque las mejoras no se centraron ya tanto en el área lógica como sucedía en el caso de la edición comentada de 1951. En la 5. a edic. (1964), Gödel goza de dos entradas: una personal "GÖDEL (KURT)», otra encabezada "GÖDEL (PRUEBA DE)» que detalla informalmente su proceder y el resultado de incompletud (1931), con la mención de sus artículos (1930) y (1931) en la bibliografía, y varias referencias complementarias. Claro está que los años 60 vienen siendo ya tiempos un tanto avanzados en la recepción de los teoremas de Gödel en España, al menos en determinados medios ilustrados filosóficos y matemáticos, y hay alguna exposición técnica alternativa a la divulgación del Diccionario. Pero, antes de los 60, aún queda bastante tela que cortar, en particular entre los filósofos.

\subsection{El despiste de un sismógrafo y los avatares del «teorema» en los años 40-50}

Entre el exilio exterior y el interior, provocado por la Guerra Civil y sus secuelas, se movió la personalidad filosófica más ilustre e ilustrada de estas déca- 
das 1930-50, José Ortega y Gasset. Conocido por sus virtudes como sismógrafo de los movimientos de ideas en Europa, Ortega tuvo noticias de las crisis de fundamentos de las ciencias (f́́sica, matemáticas, lógica) y de la quiebra de la razón, en la tradición de la filosofía occidental, que habían aireado algunos intelectuales de las primeras décadas del s. XX, entre los que él mismo vino pronto a ejercer un papel protagonista. En una conferencia dictada en Lisboa sobre la razón histórica, en 1944, tras pasar revista a las crisis de fundamentos de las ciencias, se detiene en la lógica y en el desdén de Brouwer cuando la califica de usoi-disant lógica»: "iLa lógica soi-disant! ¿Se percatan ustedes bien de la enormidad que esto significa? Porque esto significa, lisa y llanamente, que la lógica es i-lógica, por tanto que no hay lógica. Esto es lo que llamo - y no me parece exagerada la imagen- el terremoto en la razón» (en Obras completas, XII, p. 288). Esta impresión no es circunstancial. En 1941, en una contribución a Logos - revista de la facultad de Filosofía y Letras de la universidad de Buenos Aires-, declaraba:

«Desde hace tres generaciones, ha acontecido con la logicidad lo que con otros grandes temas de la ciencia... Cuando se ha querido construir lógicamente la lógica, en la logística, lógica simbólica y lógica matemática, se ha visto que era imposible, se ha descubierto con espanto que no hay concepto último y rigurosamente inédito, que no hay juicio del que se pueda asegurar que no implica contradicción, que hay juicios los cuales no son ni verdaderos ni falsos, que hay verdades de las cuales se puede demostrar que son indemostrables, por tanto ilógicas» $(O C, \mathrm{~V}, \mathrm{pp} .529){ }^{27}$.

Por aquí asoma una interpretación peculiar, un tanto tremendista, de los resultados críticos de Gödel. Años después, en el Rencontre de Ginebra (1951), la referencia se torna franca y expresa: Ortega cuenta que, hablando en cierta ocasión con un físico sobre la crisis supuesta en física por el principio de incertidumbre, él añadió por su parte:

27 En 1944, la existencia impuesta de verdades indemostrables se remacha con una cita literal de Quine (1940), Mathematical Logic: "that must always be indemonstrable mathematical truths», cf. la traducción de José Hierro de la 2.. edic. Lógica matemática (Madrid, Revista de Occidente, 1972 ; p. 24). Ortega prescinde del contexto de la frase de Quine, que pasa a explicar luego: “El descubrimiento mismo de que debe haber siempre verdades matemáticas es un descubrimiento que no podría haberse hecho sin analizar la noción de "prueba" o "teorema" explícitamente en términos de características notacionales de los enunciados" (ibd., p. 25). Este contexto podría haber indicado a Ortega que Quine y él estaban entendiendo cosas muy distintas bajo el nombre de "lógica". 
«lo que ocurre a la lógica, último y universal cimiento de nuestra civilización, es todavía más grave. El físico me miró a los ojos sorprendido, quizá, de que esté al corriente de lo que casi es todavía un secreto, gracias a su carácter sumamente abstruso y cuya formulación rigurosa es recientísima. Entonces me preguntó: "¿́e refiere usted al teorema de Gödel?». "Naturalmente. Me refiero a ese teorema que da una expresión definitiva a lo que se presentía en lógica desde hace muchos años». El teorema de Gödel significa que hablando estrictamente no hay lógica, que lo que se llama así no era más que una utopía, que se creía en una lógica aunque ésta no era desde Aristóteles más que un desideratum, un simple programa. En los últimos cincuenta años, desde Russell-Whitehead por un lado, y Hilbert por otro, se ha intentado realizar la lógica y se ha descubierto que era imposible, porque, hablando con rigor, la lógica no existe" (OC, IX, p. 663).

Es muy posible que Ortega entienda por "lógica" la tradición lógico-filosófica que la había tratado como una fundamentación de la teoría del conocimiento y la había erigido en guardián de las seguridades de la Razón y de las evidencias y certezas del Pensamiento. Es casi seguro que su impresión del "teorema» de Gödel, lejos de responder a un conocimiento directo o a un interés por su significación lógico-matemática interna, obedece al ambiente ideológico creado en parte por las paradojas, en parte por la reacción contra la "razón abstracta" lógica y científica, y sirve a los intereses de su propia alternativa filosófica, el programa de la "razón vital». Lo cierto es que su retórica induce a estar de vuelta de la lógica coetánea sin haber ido siquiera.

Si en rigor la lógica no existe, no extrañará que entre los orteguianos de rigor no haya lógicos ${ }^{28}$. No obstante, por una suerte de incongruencia pragmática, Ortega encarga a finales de los 40 a Manuel Granell la confección de un manual de lógica para Revista de Occidente - cuando el propio Granell habría preferido explayarse sobre la logicidad-. El manual se publica en 1949. Esta Lógica de Granell (1949) hace un notable y, para entonces, excepcional esfuerzo de comprensión y exposición de la nueva lógica, a la luz de Principia Mathematica y de algunas alternativas posteriores disidentes. Toca a

28 Un estudio general de las actitudes y contribuciones de Ortega y su escuela con respecto a la nueva lógica, así como su confrontación con las de la coetánea "Escuela de Barcelona", pueden verse en L. VEGA, "La suerte de la lógica en la "Escuela de Madrid»: notas sobre una desgracia", Revista de Filosofia, 28/1 (2003), 33-58. 
Gödel de pasada, en una nota a pie de página sobre el formalismo de Hilbert: menciona su artículo (1930), pero en realidad se refiere al segundo teorema de limitación de (1931), cf. o.c., Tercera parte, c. II, $\$ 1$, nota 10, pp. 283-4.

Otro caso próximo al de Ortega, en el presente contexto, es el de Xavier Zubiri. Nunca fue orteguiano, pero hizo su tesis doctoral bajo la dirección formal de. Ortega e ilustra cabalmente dos de sus querencias: [1] la insistencia inicial en la crisis del pensamiento coetáneo - cf. su diagnóstico de esa situación intelectual en Zubiri (1943) —, y [2] posteriormente, en la época de madurez de su filosofía, el uso sesgado del "teorema» de Gödel en beneficio de sus propias tesis filosóficas. Por ejemplo, a tenor de Zubiri (1982), Inteligencia y Logos, el "teorema» de Gödel no viene a significar, como suele suponerse, una limitación lógico-matemática o axiomática intrínseca; antes bien, lo que hace es respaldar una tesis ontológica, la tesis de que la realidad matemática no se agota en los postulados, y viene a poner esta realidad ante la inteligencia, pues lo real antecede a lo verdadero en la matemática (cf. o.c., pp. 138-9 y 143-6 passim).

Además de filósofos, también hubo matemáticos que sobrevivieron al trance de la Guerra tanto en el exilio exterior como en el interior. Uno de éstos últimos era José Barinaga —de quien Zubiri había recibido clases de matemáticas y a quien Granell (1949) no olvida en su bibliografía- Barinaga ya se había ocupado de algunos temas fronterizos de la lógica con las matemáticas en los años anteriores a la Guerra Civil. Había sido colaborador habitual de la Revista Matemática Hispano-Americana e, incluso, director del Laboratorio de Matemáticas - sustituyendo al patrón natural, Julio Rey Pastor-, dentro de la Junta para la Ampliación de Estudios, en cuyo marco había publicado una Miscelánea Matemática (1937) que daba cuenta de sus intereses y noticias avanzadas. Habiendo sido, en suma, un joven curioso y prometedor, labró su desgracia primero, en 1932, al contravenir los deseos de Rey Pastor en una oposición de Esteban Terrradas a una cátedra en Madrid y, luego, al quedarse en España tras la Guerra sujeto a una depuración -entre 1939 y 1946 - y a un aislamiento, de los que no llegó a recuperarse cuando fue restituido a su cátedra ${ }^{29}$. No obstante, en los años 40 , Barinaga volvió a publicar notas sobre las paradojas y misceláneas de cuestiones metamatemáticas en la revista Euclides, nacida entonces de su colaboración con Gallego

29 Véase N. CUEsTA, «Don José Barinaga Mata. In Memoriam», Gaceta Matemática, 3-4 (1966), 63-86. 
Díaz ${ }^{30}$. Discurren en este sentido dos artículos (1943), «Metamatemática y metalógica» y (1944) «Las paradojas semánticas y sus relaciones con la metamatemática», quizá las primeras publicaciones matemáticas españolas que se hacen eco de los resultados de Gödel. Su (1943) maneja una bibliografia abundante y bastante actualizada sobre el tema tratado, incluidos los trabajos de Gödel de 1930 (teorema de completud), 1931 y 1933 (teoremas de incompletud), ambos citados aunque no leídos- por sus ediciones originales. Barinaga trata aquí de recuperar unos intereses de los que ya se había ocupado antes de la contienda civil y que había expuesto en una conferencia en julio de $1936^{31}$. El artículo es un resumen del estado de la metamatemática tal como se desprende del estudio de la bibliografia extranjera manejada. Cita como cuestiones actuales fundamentales: «el problema de la decisión (Entscheidungsproblem)", «la prueba de la no-contradicción (Widerspruchsfreiheitsbeweise)" y "la completitud (Vollständigkeitsproblem)», y presta especial atención a las formas normales de «llenabilidad" (satisfacibilidad), si bien cae en el error de mencionar el trabajo de Gödel (1930) para referirse a sus resultados de incompletud (1931). Las esperanzas puestas por Barinaga en el que considera todo un nuevo campo de estudio en matemáticas son evidentes y el párrafo final del artículo nos revela su interés propedéutico: «El estado actual de

${ }^{30}$ Euclides, "Revista mensual de ciencias exactas, físicas, químicas, naturales y sus aplicaciones", se publicó en Madrid entre los años 1941 y 1960. Contenía artículos de estas materias y, aunque el Ministro de Educación Nacional, José Ibánez Martín, alabara en su $4^{\circ}$ aniversario su labor en el "campo de la matemática", la verdad es que la matemática distaba de estar omnipresente y consistía básicamente en artículos sobre geometría. Los textos de Barinaga sobre metalógica y metamatemática tenían un perfil y una presencia todavía más singulares dentro de la revista, con alguna rara e incipiente compañía como la de J.R. FUENTES MIRAS, "El problema de la decisión en el cálculo de enunciados", Euclides, 34 (1943), 669-672).

${ }^{31}$ La conferencia La verdad en la matemática, fue dictada el 2 de julio de 1936 a los aspirantes a "encargado de curso de la 2.2 enseñanza", y publicada más tarde junto con otras del propio Barinaga, de entre los años 1932-36, en BARINAGa MATA, J., Seis Conferencias, Junta para la Ampliación de Estudios e Investigaciones Cientificas, Madrid, 1938. En una nota inicial, advierte Barinaga "Sé también que el lector desconocido, al enjuiciar este folleto, ha de tener en cuenta la fecha y el lugar de la edición». Allí se comenta el carácter, menos absoluto de lo que tradicionalmente se pensaba, de la "verdad matemática", con reflexiones acerca de la crisis de fundamentos y del desafío intuicionista. Se cita, precisamente, el teorema de Goldbach como ejemplo de problema no resuelto ni soluble con los métodos matemáticos conocidos $\mathrm{y}$, finalmente, se anuncia la reciente aparición de una "nueva rama filosófico-matemática titulada METAMATEMÁTICA, que se propone discutir y aclarar los fundamentos de nuestra ciencia (...) Los nombres de Hilbert, Herbrand, Gödel, Bernays, Ackermann, Chevaley, Gentzen, Neumann, Skolem, etc., llenan ya una abrumadora literatura en plena evolución y febril actividad" (l.c., p. 57). 
estos estudios ofrece temas abundantes para monografías, tesis doctorales, cursillos sobre Metodología y concursos a premios de las entidades científicas. Con lo expuesto, queda realizado el propósito que nos ha guiado para redactar estas líneas, el cual, como hemos declarado al comenzar, no ha sido otro que ampliar un poco las ideas generales esbozadas en nuestra conferencia de 1936: "La verdad en la matemáticam". Ideas que, por cierto, en 1943 seguían sin ser escuchadas y continuaban resultando tan exóticas como innovadoras.

En el otro artículo (1944), que versa sobre las paradojas semánticas, volvemos a encontrar un interés por el desarrollo de la metamatemática y un intento de comentar los resultados de Gödel en el contexto de resolución de paradojas. Cita a este respecto la teoría de los tipos de Russell y los trabajos de Ramsey. Comenta las antinomias más conocidas: la de Epiménides - llamada en este artículo antinomia de "Lügner" («mentiroso»)-, y las de Russell, Burali-Forti, Richard, etc. Trae a colación el interés de Gödel por «encuadrar el problema dentro de los límites de representación y de inferencia de los formalismos deductivos", y al final encuentran expresión correcta los enunciados del teorema de incompletud y su corolario. Pero el artículo adolece de una confusión generalizada entre la dimensión sintáctica y la semántica - ya presente en el artículo anterior (1943) - , al referirse a la reflexividad de uno y otro tipos sin reparar en su distinción, e insinúa una más que dudosa formalización (pretendidamente gödeliana) de la paradoja del mentiroso en un "formalismo" F que supuestamente cumpla con las condiciones de representación y codificación numérica marcadas por Gödel en su prueba ${ }^{32}$. Está claro que el análisis semántico de Tarski también se estaba haciendo desear y esperar ${ }^{33}$.

32 El grado de información de Barinaga Mata en este campo era, con todo, muy superior al de otros colegas matemáticos de aquellos años, En la misma revista Euclides, 36 (1944), 189), nos encontramos con una nota del Rvdo. P. Tomás Gallarta (Misionero del Corazón de María) que pretende haber demostrado la "perfecta aplicación del método de inducción completa a los conjuntos no-numerables" por medio de una ingenua reducción al absurdo que prescinde de cualquier marco formal y que asume la consistencia (e incluso la $\omega$-consistencia) de la matemática "en general». Pero, cuando menos, el P. Gallarta también tenía deseos de aprender y aplaudirá la promoción de la asignatura "Teoría del conocimiento" en la sección de Matemáticas de la facultad de Ciencias: "Esto quiere significar — dirá con optimismo- una incorporación oficial de nuestra Universidad a los estudios logísticos casi totalmente desconocidos en nuestra Patria» ("Fundamentos racionales de la Lógica Matemática», Euclides, 49 (1945), p. 169. Sus ideas sobre esta teoría del conocimiento y su lógica subyacente son menos prometedoras.

33 Será Fuentes Miras quien dé noticia pública de su existencia: "La "verdad deductiva" según Tarski», Gaceta Matemática, VI/5-6 (1954), 110-20. Tres años antes había aparecido su 
Los años 50 marcan la reintroducción de la nueva lógica en España y el inicio de un proceso de recepción efectiva que llevará a su implantación institucional en los 70, dentro del currículo filosófico y por lo regular de la mano de la recepción pareja de la filosofía analítica. El principal nicho de acogida fue el habilitado en el C.S.I.C. por un grupo heterogéneo de personas que dio origen a la revista Theoria (1952-1955) y al Seminario de Lógica Matemática (1953), animado y orientado por su secretario, Miguel Sánchez-Mazas. En otros medios también aparecían iniciativas en análogo sentido: en el año académico 1952-3, Fuentes Miras impartía un cursillo de introducción al cálculo lógico en la Real Academia Matemática Española; en el 56-7, Manuel Sacristán iniciaba un seminario sobre lógica matemática en la facultad de Económicas de Barcelona; en el 59-60, la cátedra de Análisis Matemático de Madrid (desempeñada por Rodríguez Bachiller con Fuentes como ayudante) dedicaba un trimestre del Análisis de $4 .^{\circ}$ a impartir una introducción a la lógica de primer orden. No es extraño que las referencias autóctonas a los resultados de Gödel empiecen a hacerse con mayor precisión y conocimiento de causa - como, por ejemplo, la de Sánchez Mazas a la imposibilidad de demostrar la consistencia de un sistema que incluya la aritmética con sus propios recursos, donde hay también una mención de Gentzen (sesiones del 30 de octubre y 13 de noviembre, 1953, del Seminario de Lógica Matemática, en Teoria, II/7-8 (1953), 183) —, aunque tampoco falten las interpretaciones un tanto equívocas del alcance y la significación de este resultado de limitación véase, por ejemplo, el párrafo final sobre "el teorema de Gödel y su aplicación" de A. Díez Blanco, "El valor de las proposiciones científicas", Revista de Filosofia, XVII/65-66 (1958), 171-179, donde "el teorema" viene a ser emblemático del salto —obligado, según el autor- de la ciencia a la filosofía ${ }^{34}$.

traducción - hecha conjuntamente con T. Rodríguez Bachiller- del espléndido manual de Tarski (1940, edición inglesa), Introducción a la Lógica y a la metodologia de las ciencias deductivas, Madrid, Espasa-Calpe, 1951.

34 La inquieta, innovadora y tenaz figura de Miguel Sánchez-Mazas no debería necesitar presentación; cf., sin embargo, el homenaje a el dedicado en J. ECHEVERRIA, J. de LORENZO y L. PeNAa, eds., Calculemos ... Matemáticas y libertad. Madrid, Trotta/Universidad del País Vasco, 1996. Por su parte, Alejandro Díez Blanco era, en los 50, profesor de Filosofía de Enseñanza Media y asiduo asistente a las "sesiones científicas" de la Sociedad Española de Filosoffa, en el Instituto "Luis Vives" del C.S.I.C. Hombre abierto e ilustrado, Díez Blanco había propuesto en una sesión de mayo de 1950 la inclusión de conocimientos matemáticos y de la lógica moderna, en particular, dentro del "cuadro de los estudios filosóficos". La propuesta fue bien recibida por el presidente de la Sociedad, el P. J. Zaragüeta, quien la reorientó hacia la promoción de una 


\section{La normalización de los años 60 y sus postrimerías}

Los curiosos impertinentes que se interesaran en España por la nueva lógica, al mediar el siglo, tenían dos opciones: correr el riesgo del autodidacto, la aventura de aprender por propia cuenta sobre textos foráneos -cuyas traducciones empezarían a prodigarse-, o salir a estudiar fuera. La primera opción todavía era muy común en los años 70 . Pero un caso temprano y paradigmático de la segunda opción fue el de Manuel Sacristán Luzón; a él también le debemos nuestra primera exposición cabal de los teoremas de limitación de Gödel, digamos: su recepción normal.

\subsection{Manuel Sacristán}

En 1954, Manuel Sacristán, tras haber cursado derecho y filosofía, obtuvo una beca para ampliar estudios en Alemania y se decidió por el Institut für mathematische Logik und Grundlagenforschung de la Universidad de Münster (Westfalia), donde permaneció hasta 1956. Este instituto, fundado por Heinrich Scholz, era uno de los centros más importantes de investigación lógica en Europa. Allí logró Sacristán su competencia en esta materia estudiando con los profs. H. Hermes y G. Hasenjaeger, aunque el mentor de sus intereses lógico-filosóficos fuera el recién jubilado Scholz, por quien seguirá sintiendo especial debilidad y reconocimiento. A su vuelta a España, Sacristán comenzó a dar clases de lógica y filosofía de la ciencia en la Universidad de Barcelona con "un nivel y rigor desacostumbrados», en palabras de Jesús Mosterín. De 1956 datan unos apuntes de Fundamentos de Filosofia, editados por el SEU, en los que la lógica cubre 71 páginas de un total de 131. En 1962, acudió a unas oposiciones a la cátedra de Lógica de la Universidad de Valencia, que no obtuvo por recelos extra-académicos. Mosterín comenta a este propósito cómo "disimulaba sus "peligrosos» conocimientos de lógica matemática y citaba de memoria a Santo Tomás en latín", mientras centraba su lección magistral en el Calculus Universalis de

sección de Filosofía e Historia de la Ciencia, ya acordada. La sección se creó en diciembre de 1950 y se confió su dirección a J. Rey Pastor; en los primeros 50, contribuyó a alumbrar y alimentar la revista Theoria (1952-1955) y el Seminario de Lógica Matemática, cuyos cortos pero intensos años de vida dependieron sobre todo del entusiasmo y tesón de Sánchez-Mazas. 
Leibniz ${ }^{35}$. La opción de Sacristán por Leibniz no era táctica ni circunstancial: formaba parte de un interés temprano por lo que él llamaba «el programa algorítmico" —que ya le había llevado a redactar una contribución a un coloquio en Münster en septiembre de 1955: “Über die "Ars Magna" des Raimundus Lullus"-, interés acrecentado en el curso de sus relaciones con Scholz ${ }^{36}$. Ni que decir tiene que esta preocupación por "el programa algorítmico», reanimado y actualizado por el programa de Hilbert, también se reflejará en sus reflexiones sobre los resultados de limitación de Gödel.

Sacristán, pese a sus desventuras con el Régimen ideológico y académico en filosofía, se dedicó en los 60 a la traducción de libros capitales de Quine siendo el introductor de su obra lógica y filosófica en el español一, y a sus clases de Lógica y de Filosofía y metodología de la ciencia en la facultad de Económicas de Barcelona, hasta verse expulsado de la Universidad por disolución de su contrato en 1965. En 1962, sus apuntes ciclostilados de filosofía de la lógica mostraban una orientación lógico-filosófica del análisis lógico, afinada en Münster y enterada de las aportaciones semánticas de Carnap, Tarski, Quine, a la vez que lúcida y reflexivamente asumida. Pero, sobre todo, en 1964, publica una Introducción a la Lógica y al análisis formal, "el primer texto satisfactorio de Lógica publicado originalmente en España», según Mosterín ${ }^{37}$.

35 Merece la pena reproducir algunos comentarios de Mosterín sobre los miembros del tribunal, también indicadores de los miserables signos de los tiempos y de la situación de la lógica en España: «a todos ellos había llegado la fama de Sacristán de ser ateo, marxista, «positivista" y lógico matemático, características todas ella consideradas alarmantes, corrosivas y vitandas por el ignorante y mojigato mundillo filosófico oficial de la España de entonces, dominado por curas, semicuras, metafísicos tomistas y paletos reaccionarios de diversa laya (...) Más misterioso era el pánico que sentía el establecimiento filosófico académico por la Lógica Matemática (...) la temían, en parte, por ignorancia y miedo a lo desconocido, y en parte porque sospechaban que el mero contacto con algo tan claro y preciso podría hacer reventar el endeble entramado filosófico neomedievalizante que servía de soporte al nacionalcatolicismo oficial de la época» [prólogo a Sacristán (1996), p. vi].

36 Véase Albert Domingo CuRTo, "Manuel Sacristán y el estudio de la obra lógica de Leibniz", en S. López Arnal y otros, El valor de la ciencia, Barcelona, El Viejo Topo, 2001, 21334 , con un anexo de fragmentos de estudios inéditos de Sacristán sobre el "Calculus" y sobre la identidad de los indiscernibles, 235-48.

37 Mosterín hace en el prólogo recién citado un breve repaso a los textos de Lógica anteriormente publicados en España o en espańol, alguno ya nombrado en este trabajo: el de García Bacca de 1936 ("su nivel científico dejaba bastante que desear"), el de Ferrater Mora-Leblanc, México, 1955 (“de merecida repercusión (...) su presentación de los cálculos deductivos era insuficiente y no permitía aprender a deducir") y el de Miguel Sánchez Mazas, Fundamentos 
En este texto aparece la primera exposición precisa y cumplida, aun si informal y perfectamente legible, de los teoremas de limitación de Gödel en España, dentro de un contexto de presentación general de la lógica formal que no elude ni consideraciones filosóficas, ni temas complementarios como la definición o la inducción. Ya en la primera parte, titulada "La lógica formal y las ciencias reales. Categorías Lógicas", nos encontramos con el Cap. 3, «El ideal del lenguaje bien hecho" en el que se presentan las propiedades metalógicas de los cálculos: la consistencia, la completud y la decidibilidad. En el parágrafo $\$ 20$, «Los límites del ideal algorítmico», Sacristán señala: «se pensó durante algún tiempo que el programa algorítmico fuera plenamente realizable (...) Pero a partir de 1930 varios autores demostraron que esa suposición era excesiva (...) sólo puede reducirse de un modo general a algoritmo una parte de la lógica que no llega al grado de complicación de la aritmética" (o.c., p. 53). En el $\$ 21$, "Los frutos del programa algorítmico", añade:

"Una empresa verdaderamente científica no es nunca estéril, aunque no alcance nada de su objetivo inicial. Así ocurre con el programa algorítmico, el cual, por lo demás, consigue algo relacionado con su objetivo, a saber, un considerable aumento de la potencia deductiva de la lógica, un enriquecimiento del arsenal de los métodos formales» (ibd.).

Entre estas coordenadas se va mover la interpretación de Sacristán de los teoremas de limitación de Gödel (1931): entre la renuncia a la utopía algorítmica de una reducción de toda inferencia deductiva a un cálculo y de la resolución por esta vía de cualquier debate o problema teórico, y el reconocimiento del poder adquirido por unos métodos lógicos formales capaces de demostrar sus propios límites. Luego, dentro de estas coordenadas, habrá intérpretes de Sacristán que difieran acerca de la tendencia de sus movimientos: según Sánchez-Mazas, se acercará demasiado a la ordenada crítica, por prestar mayor atención a las pretensiones de "autojustificación" de los formalismos que a su

matemáticos de la lógica formal, Caracas, 1963 («que tuvo menos difusión y presentaba un punto de vista intensional poco estándar"). "Por todo ello, sólo a partir de 1964, después de la publicación en Barcelona de Introducción a la Lógica y al Análisis formal de Manuel Sacristán, dispusieron los estudiantes españoles de un libro de texto de Lógica riguroso, útil y a la altura de las circunstancias" (l.c., pp. vi-vii; anotemos que el libro mentado de D. García Bacca, Introducción a la lógica moderna, Barcelona, Labor, 1936, "no es un tratado de logística [o de lógica moderna]; es una meditación fenomenológica de la lógica entera", según su propio autor, o.c., p. 31 ). 
capacidad expresiva y su potencia analítica; según Mosterín, tenderá más bien a la abscisa utópica, a preocuparse por el modo de salvar esas limitaciones ${ }^{38}$. La verdad es que los comentarios de Sacristán al respecto, en el $\$ 68$ «Sobre la significación del teorema de incompletud de Gödel para la teoría de la ciencia” (1964, pp. 197-9), parecen equidistantes de una y otra. Pasemos, pues, a su propia presentación de los tres teoremas capitales de Gödel.

El teorema de completud del cálculo lógico de primer orden es la culminación ( $\$ 63$, pp. 183-5) del capítulo XI, dedicado al rendimiento del cálculo lógico elemental. Su exposición no sigue a Gödel (1930) sino más bien el tratamiento simplificado de Hilbert-Ackermann (1949 3. ${ }^{\mathrm{a}}$ edic.) ${ }^{39}$. Los teoremas de incompletud de Gödel (1931) se presentan en el capítulo siguiente, que se inicia con una noticia de la lógica de orden superior; en este caso, Sacristán opta por asumir la revisión informal de J.B. Rosser (1939) ${ }^{40}$ adoptada por Nagel y Newman (1958). La prueba del teorema de incompletud viene precedida de un parágrafo dedicado a la gödelización $(\$ 66)$, en el que se desliza este párrafo: «lo esencial de la gödelización es esto: la lógica de predicados (de orden superior) permite expresar la aritmética (...) en ella pueden también expresarse afirmaciones acerca de las afirmaciones aritméticas: por ejemplo, que tal afirmación aritmética es verdadera of falsa o que es demostrable o que es indemostrable» (p. 191; cf. también la p. 192, en la que se insiste en la expresión aritmética de expresiones metaaritméticas sobre la verdad, falsedad, demostrabilidad o indemostrabilidad). El desliz estriba en no distinguir entre los predicados metateóricos sintácticos "demostrable / indemostrable" y los semánticos "verdadero / falso": la verdad (falsedad) en la aritmética no puede definirse dentro de la aritmética, como había mostrado Tarski o cabía inferir de la paradoja del "mentiroso», mientras

38 La opinión de SÁNCHEZ-MAZAS se encuentra en una carta a Sacristán (8 de enero, 1965), que saluda su (1964) Introducción a la lógica y al andilisis formal con entusiasmo y espera que contribuya al saneamiento y a la regeneración de la cultura lógica del país. La opinión de Mosterín puede verse en la entrevista recogida en S. López Arnal y Pere de la Fuente, eds. Acerca de Sacristán, Barcelona, Destino, 1996, p. 662, donde también asegura que Sacristán «era una de las pocas personas que conocía y entendía bien el teorema de Gödel", de modo que así mismo le preocupaban las apelaciones absurdas u oscurantistas al teorema para descalificar trabajo o el valor de la ciencia (pp. 662-3).

39 Poco antes había aparecido la traducción de su $4 .^{2}$ edic.a cargo de Víctor SANCHEZ DE ZaVala: D. Hilbert y W. Ackermann, Elementos de lógica teórica, Madrid, Tecnos, 1962.

40 Véase la traducción de L. VEGA DE J.B. ROSSER (1939), «Una exposición informal de los teoremas de Gödel y Church», en P. Castrillo y L. Vega, eds. Lecturas de Lógica II, Madrid, UNED, 1984, 362-74. 
que la demostrabilidad (indemostrabilidad) en la aritmética sí puede definirse dentro de ella, como muestra la aritmetización gödeliana. De ahí resulta que si todas las fórmulas demostrables del sistema son verdaderas, ha de haber alguna fórmula verdadera pero indemostrable, es decir: una vía de acceso a la incompletud como insuficiencia -que no deja de inducir a Sacristán a algún equívoco- No obstante, Sacristán expone a continuación $(\$ 67)$, con bastante detalle y mucho cuidado en el uso de los niveles lingüísticos y metalingüísticos, la prueba de Gödel y la construcción de su fórmula indecidible, para evitar su confusión con la paradoja del mentiroso (o "de Epiménides»); declara incluso:

"Es muy importante comprobar que (11) [i.e. la fórmula aritmética que en su interpretación metalingüística natural afirma un caso de no demostrabilidad] no tiene el defecto de estructura del enunciado causante de la paradoja de Epiménides (...), pues no afirma de sí misma que es falsa, ni que es indemostrable. Lo que afirma, como fórmula aritmética que es, es que no hay ningún número, $\mathrm{x}$, que esté en la relación aritmética $\mathrm{D}$ con el número $\mathrm{m}$. Y como la relación $\mathrm{D}$ es aritmética, aquí no hay ninguna mezcla de niveles lingǘsticos, como en la paradoja de Epiménides" (p. 196).

También tiene especial importancia el comentario que el autor dedica en el parágrafo siguiente $(\$ 68)$ a algunas «interpretaciones» espurias del teorema, como las que pretenden que Gödel ha demostrado "el fracaso de la lógica" o hasta "el fracaso de la razón». Contra semejantes posturas presenta cuatro consideraciones: 1) La imposibilidad de conseguir un conjunto de axiomas y un juego de reglas de deducción que suministren todas las expresiones verdaderas en el lenguaje de la lógica de enunciados no excluye que los criterios lógico-formales en particular, y los criterios racionales en general, sigan valiendo, al igual que no cabría acusar a la prueba misma de Gödel, que evidencia dicha imposibilidad, de ilógica o irracional; lo que se desprende de esta limitación de un sistema formal es que no se contiene ni se justifica totalmente a sí mismo, condición en la que conviene con la práctica racional de las ciencias. 2) El propio descubrimiento de Gödel es un éxito de la lógica, es la propia lógica la que ha descubierto los límites o la inviabilidad del programa algorítimico en su forma clásica: la que ha que permite saber cuáles de sus actividades son algoritmizables y cuáles no. 3) La incompletud de un cálculo tomado en toda su dimensión no excluye la completud de algunos sistemas parciales contenidos en él; por otra parte, también puede conseguirse cierta completud del cálculo de predicados general, aunque al precio de su interpretación no estándar, según ha establecido Henkin (1950). 4) Los enunciados cuya 
indemostrabilidad establece la prueba de Gödel "no son de mismo estilo" que las proposiciones clásicas de la matemática, referentes a operaciones con números y aplicadas realmente en nuestras labores científicas o técnicas; para esta parte "útil" de la matemática se han construido sistemas que dan de sí todos los teoremas interesantes (pp. 198-9). Dentro de esta perspectiva bastante razonable, puede que no sean impertinentes un par de observaciones: en principio, dado el interés de Sacristán por el programa algorítmico, es curioso que no preste mayor atención a las funciones recursivas también introducidas por Gödel (1931); por otro lado, con respecto a su consideración en el punto 4), el paso del tiempo y el desarrollo de las líneas de investigación abiertas por Gödel han llevado a conocer proposiciones matemáticas indecidibles más normales, que no exigen una codificación gödeliana, según han demostrado Paris y Harrington en 1977. Pero este resultado, a la par que alguna consideración avanzada en 1), también nos remite al otro teorema de limitación de Gödel (1931), a la imposibilidad de demostrar la consistencia de la aritmética con los recursos finitistas propios del sistema considerado. Sacristán esboza de modo claro y sucinto su prueba en el parágrafo $\$ 69$, pp. 199-200, y en el contexto de una confrontación con la versión moderna del viejo ideal algorítmico, el programa de Hilbert.

En fin, no hace mucho se ha publicado un libro póstumo, Lógica elemental, que responde - salvo correcciones menores - al borrador que Manuel Sacristán había preparado hacia 1965-66 para una enciclopedia temática proyectada por la Editorial Labor. Tiene una hechura evidente de manual de iniciación a la lógica, más sencillo y amistoso que su Introducción (1964) ${ }^{41}$, e incluye una bibliografía específica en cada capítulo. Una característica notable de este manual es su remate con un "Esquema de Historia de la Lógica» (pp. 297-347), que pasa revista a los diversos desarrollos de la lógica, desde su matriz griega hasta el presente. Sacristán utiliza ya la moderna historiografía oficial: los textos de Bocheński (1951, 1956), Lukasiewicz (1951), los Kneale (1962), amén de reconocer figuras entonces en la sombra, como la de Peirce ${ }^{42}$. En el

41 En respuesta de 5-X-65 a una carta de Ferrater Mora en la que éste calificaba de "excelenten su Introducción a la Lógica..., Sacristán da a entender las dificultades que este texto suponía para sus alumnos de primer curso.

42 Aunque, naturalmente, no pueda prever desarrollos sustanciales de la historiografía de la lógica como los que han tenido lugar a partir de los años 1970, la visión histórica de Sacristán no tiene el aire convencional de un mero apéndice de oficio, añadido para dar lustre a un manual y "cultureta" a los estudiantes de lógica. 
apartado histórico dedicado a Gödel, Sacristán hace breve recordatorio del teorema de incompletud, tras el que recoge las consideraciones ya opuestas en la Introducción a la Lógica $(\$ 68)$ a sus versiones catastrofistas; luego resume el segundo teorema de limitación, acerca de la indemostrabilidad de la consistencia de la aritmética por medios finitistas, para concluir que si bien todo esto «destruye el programa de Hilbert tomado al pie de la letra", "no muestra la esterilidad de ninguna de las ideas básicas del formalismo» (p. 345). Por lo demás, a su juicio, el teorema de incompletud no anula el programa logicista de Russell (p. 344).

En estos textos de Sacristán nos encontramos ya con cierta situación de normalidad en el tratamiento de la materia lógica, normalidad todavía carente de un respaldo institucional que luego iría adquiriendo con el avance de los 60 y en el curso de los 70 - al menos en las facultades de filosofía y de la mano, sobre todo, de la filosofía analítica-. Evidentemente estamos ante manuales introductorios, un tanto eclécticos en el uso de métodos lógicos, con una semántica suplantada por glosas informales y más sugerentes en el plano filosófico que en el plano técnico. Ahora bien, todo esto, dada la falta de información que existía en el ambiente, fue seguramente una ventaja que facilitó la ilustración lógica de los relativamente numerosos lectores de su Introducción ..., dentro y fuera del recinto académico de la filosofía y de la metodología de la ciencia en Barcelona.

\subsection{Postrimerías de la recepción y sugerencias para un balance provisional}

Llegados a Sacristán (1964) parecería que habríamos acabado nuestro recorrido: la nueva lógica matemática y con ella los resultados de Gödel pasaban a integrarse en el moderno cuadro de conocimientos que se habrían de estudiar a partir de entonces en España. Pero aún deberemos mencionar dos últimos casos, diametralmente opuestos entre sí, que forman parte de nuestra recepción de los teoremas de limitación de Gödel (1931). El primer caso es uno de los episodios más singulares de toda esta historia: la irrupción de los «refutadores», a finales de los 60 y principios de los 70. La sucesión de refutaciones de las filosofías modernas es una larga, si no brillante, tendencia histórica de nuestra filosofía académica por los ss. XVIII-XX. Lo que ya no ha sido tan frecuente ha sido la refutación de teoremas. Sin embargo, algunos resultados de la lógica matemática moderna han vuelto a alimentar el hábito de la confutación; entre ellos, en particular, las 
pruebas y los teoremas de limitación de Gödel ${ }^{43}$. Así, en una comunicación al XIV Congreso internacional de Filosofia en Viena, $19688^{44}$, Jorge Pérez Ballestar presentaba unas objeciones que cabría resumir en un cargo de incompetencia lógica de Gödel (1931): la prueba parece adolecer de cierta contradicción interna (l.c., pp. 50, 51-2), la fórmula indecidible está mal construida (p. 55), hay un uso ilegítimo de la función de sustitución (p. 55). En 1972, bajo esta inspiración, Emilio Díaz Estévez lee su tesis doctoral en la Universidad del Opus de Pamplona, "La refutación del teorema de Gödel», refutación que extiende a la exposición de S.C. Kleene (1964), Introduction to Metamathematics. En un extracto mecanografiado, Díaz Estévez asegura haber hallado una contradicción entre las premisas del teorema de incompletud de Gödel (1931), y demostrado la imposibilidad de construir una proposición indecidible en los términos gödelianos; tres años después redondea y publica estos hallazgos en un libro (1975), El teorema de Gödel, que algunos tomarían entonces, conforme a su título, por una exposición o explicación de la prueba ${ }^{45}$. Díaz Estévez (1975), algo más ilustrado en este terreno que Pérez Ballestar — gracias sobre todo a la revisión enciclopédica de Ladrière (1958) —, corrige algunas apreciaciones de su maestro para coincidir en la acusación principal: la prueba de incompletud de Gödel tiene una constitución antinómica e ilegítima - pareja a la de cualquier razonamiento contaminado por el método cantoriano de diagonalización-, e incurre en cierta circularidad, incompatibilidad interna y malformación. Es una lástima que la primera monografía española sobre "el teorema» de Gödel insista en unos prejuicios y confusiones como los que habían acosado al "teorema" cuarenta años antes. La saga puede cerrarse con un artículo del P. Roig Gironella (1977): «El teorema de Gödel y la analogía del ser. Un capítulo de filosofía del lenguaje», cuyos despropósitos desafían ya la imaginación del discreto lector ${ }^{46}$.

43 Hoy mismo, en la red de Internet, puede visitarse un lugar hispánico a cargo de un psicoterapeuta en el que se refutan, además de los teoremas de Gödel, el procedimiento de diagonalización de Cantor y las bases de la teoría de la computabilidad (Turing, Church), entre otros supuestos y resultados lógico-matemáticos.

44 "Undecidability and reflexive substituions", en Akten des XIV Intern. Kongresses für Philosophie, Wien: Herder, 1969; t. III, pp. 49-56.

45 Puede verse un extracto de 27 pp. en la Biblioteca Nacional (Madrid), R. 590.900; el libro apareció en Pamplona, Ediciones de la Universidad de Navarra S.A., 1975.

46 Publicado en Espiritu, XXVI/76 (1977), 121-132, quiere ser un comentario de Díaz Estévez (1975). A Roig Gironella no parece importarle si Díaz Estévez ha conseguido refutar a Gödel o no; el asunto carece de relieve porque, en todo caso, en lógica y aritmética, empleamos lenguajes "unívocos", capaces de expresar tan sólo un nivel de realidad. Todo ello —en particu- 
Pero, por fortuna, la normalización de los estudios lógicos no progresaba en vano. $Y$ así, en el extremo opuesto, con los años 80, aparecen otras dos muestras postreras de la recepción de Gödel. La primera es la traducción española de su artículo clásico de 1931, Sobre proposiciones formalmente indecidibles de los Principia Mathematica y sistemas afines (Valencia, Cuadernos Teorema [8], 1980), a cargo de Manuel Garrido, Alfonso García Suárez y Luis Manuel Valdés; incluye la nota añadida por Gödel en 1963 y viene precedido de la "Introducción» escrita por R. B. Braithwaite para la edic. inglesa (1962). La segunda es la edición española a cargo de J. Mosterín de K. Gödel, Obras completas (Madrid, Alianza, 1981), que recoge sus publicaciones en vida. Dentro de sus ligeras diferencias - la primera versión discurre más pegada al original, la segunda se permite algunas licencias de unificación y actualización-, representan el acto final de la entretenida recepción de estos resultados lógico-matemáticos de Gödel en español.

Ensayemos ahora un balance, siquiera sea provisional. El marco internacional de recepción de los artículos de Gödel (1930) y (1931) contaba con dos dimensiones: una, filosófica, cruzada por los problemas de fundamentos matemáticos y las paradojas lógicas y matemáticas, a la que se había venido a sumar la nueva crisis anunciada por Weyl (1921) en nombre del intuicionismo y de un constructivismo no predicativo; otra, técnica, marcada por los desarrollos del programa de Hilbert y el influjo del grupo de Göttingen, sobre la base del sistema dominante de Principia Mathematica. En España, a principios de los años 30, había noticias de ambas, en especial de la primera. Pero no se conocían directamente los resultados de Gödel, según se desprende entonces $-\mathrm{y}$ aun años después- de las confusiones entre ambas publicaciones de Gödel (1930) y (1931), y de las referencias un tanto imprecisas al teorema de incom-

lar, el conjunto de problemas y de paradojas consiguientes- se superaría con el reconocimiento de la Analogía escolástica y, en particular, con la admisión de un lenguaje primero autofundante, que contuviera en sí la referencia a una ontología total, a la suprema noción analógica del ser: "Podríamos resumir nuestro pensamiento precedente con estas palabras: si no se admite el teorema de Goedel, es preciso recurrir a la Analogía del Ser para fundamentar coherentemente el Lenguaje; si se admite la teoría de Goedel, también es preciso; por consiguiente, de todos modos es preciso recurrir a esta última fundamentación" (p. 126). Es una suposición que, a fin de cuentas, viene a constituir la conclusión. 
pletud. Para colmo, esas primeras noticias tendieron a disiparse - salvo en algún caso singular - como resultado de la Guerra Civil y su desenlace nacional-católico-(neo)escolástico. Por otro lado, los ecos de la famosa "crisis de fundamentos" y de las paradojas invitaban a interpretaciones sesgadas del "teorema», sobre todo en medios filosóficos dados a hallar "pruebas y confirmaciones» de sus presunciones previas. El tópico de las paradojas, en particular, llegaría a producir efectos contrarios -y ninguno de ellos bueno, a la luz de las confusiones que los acompańaban-: unos, amplificadores, que en los años 40 magnifican el teorema como síntoma o como ingrediente de una crisis de la razón lógico-matemática moderna y, en consecuencia, de un «modo de pensar" coetáneo; otros, reductores, que mucho más tarde asimilarán la prueba de Gödel (1931) a una muestra de antinomia o de malformación, y esto dará pábulo a las refutaciones del teorema. Gracias a algún ilustrado que tuvo que buscarse fuera su competencia en lógica, las aguas volvieron a su cauce en el curso de los años 50-60: así, cuando empezaba a normalizarse el estudio de la lógica -aunque todavía en una situación marginal y precaria, sin reflejo, diríamos hoy, en el "currículo»-, los teoremas de Gödel encuentran una exposición clara y pertinente, una presentación de cierta solvencia. Por fin, a principios de los 80, el lenguaje español también acoge la versión de esos artículos de Gödel de modo que pueden ya darse por efectivamente recibidos sus famosos teoremas en España.

\section{Referencias Bibliográficas}

BARINAGA MATA, José: “Metamatemática y metalógica», Euclides, 3/23 (enero 1943), 15-21.

- "Las paradojas semánticas y sus relaciones con la metamatemática", Euclides, 3/35 (enero 1944), 62-65.

DaWson, John W.: «Completing the Gödel-Zermelo Correspondence», Historia Mathematica, 12 (1985), 66-70.

- "The Reception of Gödel's Incompleteness Theorems", en Thomas Drucker (ed.) Perspectives on the History of Mathematical Logic, Boston, Birkhäuser, 1991, pp. 84-100.

- Logical dilemmas: the life and work of Kurt Gödel, Wellesley (MA), A.K. Peters, 1997.

Ferrater Mora, José: Diccionario de Filosofia, La Habana, 1941 (1. a ed.); Santiago de Chile-México, Atlante, 1944 (2. ${ }^{\mathrm{a}}$ ed.); Buenos Aires, Editorial Sudamericana, 1951, 1956, 1964 (3. $.^{\mathrm{a}} 4 .^{\mathrm{a}}$ y $5 .^{\mathrm{a}}$ ediciones respectivamente). 
- \& Hugues Leblanc, Lógica Matemática, México, FCE, 1955 (1962, 1965).

GARCIA BACCA, Juan David: Assaigs moderns per a la fonamentací́ de les matemàtiques, Barcelona, Institut d'Estudis Catalans, 1933.

- Introducció a la Logística, Barcelona, Institut d'Estudis Catalans, 1934 (2 vols.).

GÖDEL, Kurt: Colleted Works [edic. dirigida por Solomon Feferman]. New York 1 Oxford, Oxford University Press, 1986 (vol. I, Publications 1929-1936), 1990 (vol. II, Publications 1938-1974), 1995 (vol. III, Unpublished Essays and Letters).

- En la red puede verse un repertorio de materiales inéditos - en su mayor parte de los años 1930-1970 - preparado por J.W. Dawson y revisado por R. Schoff y V. Volz: http://liweb.princeton.edu/libraries/firestone/rbsc/godel.htm

Granell, Manuel: Lógica. Madrid, Revista de Occidente, 1949.

LADRIËRE, Jean: Les limitations internes des formalismes. Louvain, Nauwelaerts, 1957 (Trad. de J.L. BlasCO. Limitaciones internas de los formalismos. Madrid, Tecnos, 1969.)

Mancosu, Paolo: «Between Vienna and Berlin: The immediate reception of Gödel's incompleteness theorems", History and Philosophy of Logic, 20 (1999), 33-45.

Ortega y Gasset, José: Obras completas. Madrid, Alianza / Revista de Occidente, 1983 (12 vols.); hecha sobre la base de una edición anterior [Madrid, Revista de Occidente, 1946-1969, 11 vols.], esta edición tampoco llega a ser completa ni, desde luego, crítica.

SACRISTÁN LUZON, Manuel: Introducción a la Lógica y al análisis formal. Barcelona, Ariel, 1964.

- Lógica Elemental. Barcelona Vicens-Vives, 1996 (edic. póstuma).

Van Heijenoort, Jean (ed.): From Frege to Gödel. A Source Book in Mathematical Logic, 1879-1931. Cambridge (MA), Harvard University Press, 1967.

ZUBIR, Xavier: Naturaleza, Historia, Dios. Madrid, Editora Nacional, 1943.

— Inteligencia y Logos. Madrid, Alianza / Fundación Xavier Zubiri, 1982. 